\section{Haro, Guillermo. New planetary nebulae and emission objects in the region of the galactic center.}

In an area of about 600 square degrees and centered in the region of the galactic center there have been found 437 objects with strong $H \alpha$ in emission, from which I 2 I correspond to the given positions of planetaries previously discovered, 67 are classified as new planetaries and 48 as possible planetaries, or in few cases, as bright nebulae. A glance at the apparent distribution of these planetaries convinces one that these are highly concentrated towards the galactic center with a marked tendency of symmetrical distribution with regard to the galactic plane. Analogy suggests that the three dimensional system of these planetaries may be coexistent and concentric with the nucleus of our galaxy. If this is true, then the planetary nebulae may provide us with an independent means to determine the center and the probable dimensions of the nucleus of our stellar system.

National Astronomical Observatory, Tacubaya, Mexico.

\section{Herget, Paul. Coordinates of the sun.}

The coordinates of the sun have been computed at intervals of four days for the igth and 2oth centuries. The values are based on Newcomb's tables, except that a correction $+4.78 T$ to the motion of the perihelion has been introduced.

These results will be used primarily to compute the gravitational attraction of the earth-moon system upon other objects in the solar system; and secondly, for the reduction of observations of other objects from the earth. In the latter case the lunar terms are included, so that the geocentric coordinates are obtained; and second differences are printed to facilitate interpolation. In the former case, the lunar terms are not included, and the barycentric coordinates are given. To allow an interval of ten days to be used, the results have been interpolated to halves to provide the values at the odd multiples of ten Julian days.

All of the computations have been performed on standard IBM punch-card machines and the results will be published in the Astronomical Papers of the American Ephemeris. This project has been supported in part by the Office of Naval Research.

The Cincinnati Observatory, Cincinnati, Ohio.
Herrick, Samuel. A differential correction for the methods of Lagrange and Gauss-Gibbs.

The method of Gauss-Gibbs we may characterize as having the same first approximation as that used in the well-known Merton-Veithen modification of Gauss's method, but as having a final determination of the elements that is based purely geometrically upon three heliocentric positions. Gibbs's celebrated expressions for the ratios of the triangles I have equipped with a remainder term and removed to the "representation." His interesting, though primitive, differential correction I have extended to the ratios of the triangles, instead of stopping as he did with the coefficients in his expressions therefor. This simple device reduced by three-quarters his work on Comet $\mathrm{I} 88 \mathrm{oV}$.

The method of Lagrange may be defined as using also the foregoing first approximation together with some means of obtaining the velocity components for the middle date, so that it is possible to proceed with the improvement of the orbit and the final determination of the elements as in the Laplacian methods, especially that of Leuschner. It is also possible, however, to correct differentially the ratios of the triangles $c_{1}$ and $c_{3}$, as for the Gibbsian method above, after "representing" them by

$$
c_{1}=\frac{g_{3}}{f_{1} g_{3}-g_{1} f_{3}}, c_{3}=\frac{-g_{1}}{f_{1} g_{3}-g_{1} f_{3}},
$$

with values of $f_{i}$ and $g_{i}$ used in the representation of the observations.

To appreciate the proposed differential correction we may note that the Leuschner differential correction may be expressed in the equations

$$
\left.\begin{array}{l}
f_{x i} \Delta \rho_{2}+g_{i} \Delta \dot{x}_{2}=\lambda_{i} \Delta \rho_{i}+\rho_{i} \Delta \lambda_{i}, \\
f_{y i} \Delta \rho_{2}+g_{i} \Delta \dot{y}_{2}=\mu_{i} \Delta \rho_{i}+\rho_{i} \Delta \mu_{i} \\
f_{z i} \Delta \rho_{2}+g_{i} \Delta \dot{z}_{2}=\nu_{i} \Delta \rho_{i}+\rho_{i} \Delta \nu_{i}
\end{array}\right\} i=\mathrm{I}, 3,
$$

from which $\Delta \rho_{2}, \Delta \dot{x}_{2}, \Delta \dot{y}_{2}, \Delta \dot{z}_{2}$ may be determined by numerical elimination. Now these equations are not only derived from, but closely resemble in form, the equations

$$
f_{i} x_{2}+g_{i} \dot{x}_{2}=x_{i}=\lambda_{i} \rho_{i}-X_{i}, \quad i=\mathrm{I}, 3,
$$

etc., which are also basic to the fundamental equations of the Gaussian, Gibbsian, and Lagrangian methods. It is obviously desirable to use in the differential correction the already existing scheme of elimination equations based upon equations (3) in place of those that would have 